\title{
Traffic Light Control System Using Microcontroller
}

\author{
Rasha falah kadhem \\ Rasha. kadhem@qu.edu.iq
}

Al-Qadisiyah University, College of Computer Science and Information

Technology, Department of Multimedia

Received:- $\quad 20 / 8 / 2017$

Accepted:-23/1/2018

\begin{abstract}
:
Computing machines were always consided an attraction for the automation field of technology in both industrial and domestic products. For that reason, we should learn how to programed and used Microcontroller as a useful device. That led to more and more universities and colleges become interesting and starting to teach the microcontroller, especially Microchip PIC microcontrollers. This research an effort to design, programming and build a basic programmable traffic light control device for 4 tracks intersection.
\end{abstract}




\section{Defining of the Research:}

Creating a basic traffic signal for four traffic track intersection, three signals per track green, yellow and red, using microcontroller to get outputs of five volts at specific times according to the data specified in the program, which will be used to run the LEDs that represent the different signals for each track.,

\subsection{Preface and Research Importance:}

Computing machines were always consided an attraction for the automation field of technology in both industrial and domestic products. it is one of the

the computing machines.

microcontrollers had became an integral part of all automatic and semi-automatic machines, especially Microchip PIC. Remote controllers, hand-held communication devices, dedicated controllers that use it, have certainly improved the functional, operational and performancebased specifications. The architectural changes in instrumentation and control systems were and are dueto the computing and communication

\subsection{Research Objectives:}

to learn how to programming and using Microcontroller as a powerful objectives of motivation to develope the computing machines and devices. but it change after invention the first microprocessor, it was hardly to practically think of any of the algorithms in reality to use because of the size and power consumption. These limitations have been ended because the availability of powerful microprocessors and microcontrollers. smaller size and fast implementation and power consumption with effective computation are some of the issues, which could further increase expectations from

capability of the microcontroller devices. For that reason, in the past few years, more universities and colleges became interested and started to teach microcontroller, especially the Microchip PIC microcontrollers. Microchip designs and manufactures several families of 8-bit microcontrollers. Among them, the PIC 16 family and the $\mathrm{P} 1 \mathrm{C} 1 \mathrm{H}$ family are the two most important families. The PIC 18 family was the Latest development and has several advantages over the PIC microcontroller family." device, we chose to design and build a basic traffic signal for four tracks traffic intersection, three signals per 
track, green, yellow and red, using microcontroller PIC16F84A to get five volts outputs in a specific times according to the data specified in the program, which will be drive twelve LEDs that represent the different signals for each track.

\section{2- Theoretical Framework:}

\section{2-1 Microcontroller vs}

Microprocessor:

Although processors are of great importance in digital-based systems, but they are relatively bag in size that are difficult to embed in the devices that we need to control, and

It applications can be designed and built quickly and cheaply. A working system can contain of a microcontroller chip and just a few external components for feeding data and signals IN and OUT. They tend

\section{2-2 Microcontroller PIC:}

PIC Microcontroller was originally a design of the company General Instruments. It was intended for simple control applications, the name - Peripheral Interface Controller. In the 1970s, General Instruments produced the PIC 1650 and 1655 processors. Although the design was comparatively crude and unconventional, it was completely stand-alone, and contained some important and forward-looking features. The simple CPU was a RISC structure, with a single Working they have high capabilities that we often do not in need to for devicecontrol purposes, which makes it very energy-consuming. These issues make using microprocessors for automation purposes is limited, which has led to design the microcontrollers as a tool beyond these limitations to make automation of types of device and equipment more efficiency.

The microcontroller provides, in a simple form, all the main elements of the microprocessor system on a single chip. As a result, less difcu

to be used for control operations requiring limited amounts of memory but operating at high speed, with external hardware attached only as required by a specific application.

register and just 30 inst. The output pins could source or sink much more current than most other microprocessors of the time. Already the trademarks of the PIC were emerging - simplicity, standalone, high- speed and low cost.

the 1990s, the range of available PIC microcontrollers grew, and as they did so, they gradually overtook many of their better-established competitors. In many cases, PIC microcontrollers could run faster, needed a simpler chipset and were quicker to prototype with than their competitors. Unlike many competitors. Microchip made their development tools simple and low 
cost or free. Moreover, Microchip stayed firmly entrenched in the 8-bit

\subsection{PIC16F84A Microcontroller:} PIC16F84A microcontroller, along with its direct predecessors, was one of many PIC success stories. First introduced as 16C84. While most decision to stay small, simple and easy to use. While many of the microcontrollers of the day were on the wafer program on the wafer, it was usually an erom (erasable programmable readonly memory), with the attendant time-consuming errom erasing cycle. With $16 \mathrm{C} 84$, the chip chose to use the eProm (erasable electrically programmable read-only memory) for program memory.

\subsubsection{PIC16F84A Microcontroller} Architecture:

Microcontroller 16F84A is simple in its architectural design and pins layout, in order to simplify its applications. A look at its block diagram we can see that internal components essentially consist of a simple microprocessor core, along with all necessary data and program memory. To this, it adds all the peripherals that allow it to do the world.

microcontroller manufacturers are trying to make their products bigger, more sophisticated and more complex, the chip looks like a bold

Thus, it can be programmed quickly, change repeatedly. Then, as Flash memory technology became more accessible, the "C84 was reissued as 16F84, with new memory technology.With further optimization, I6F84A became .For these reasons, the researcher chose to use the PICI6F84A microcontroller in this project.

interfacing it needs to do. These may include digital and analog input and output, or counting and timing elements. Like any electronic circuit, the microcontroller needs to be powered and needs a clock signal (which in some controllers is generated internally) to drive the internal logic circuits.

\subsection{2: Block Diagram: A block}

diagram of the device is shown in

Fig.1: 


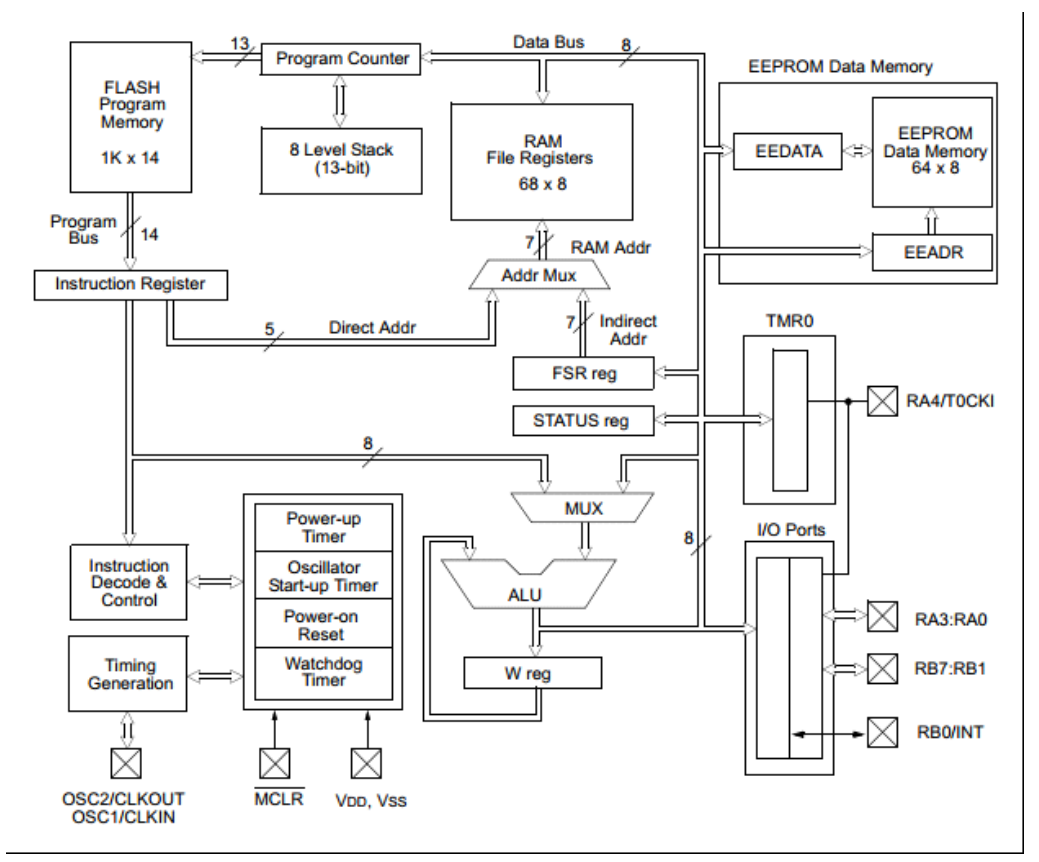

Fig 1: PIC16F84A Microcontroller Block diagram.

Where the essential parts are:

- microprocessor core.

- program memory of $1 \mathrm{kB}$, which translates to 1024 instructions

- Data memory (RAM) of 68 bytes.

- Data EEPROM of 64 bytes.
- Registers.

- Arithmetic and Logic Unit (ALU).

- Timer.

- 1/O ports.

2.3.3 Pins Layout and Functions: Fig. 2 shows 16F84A pins layout in PDIP package.

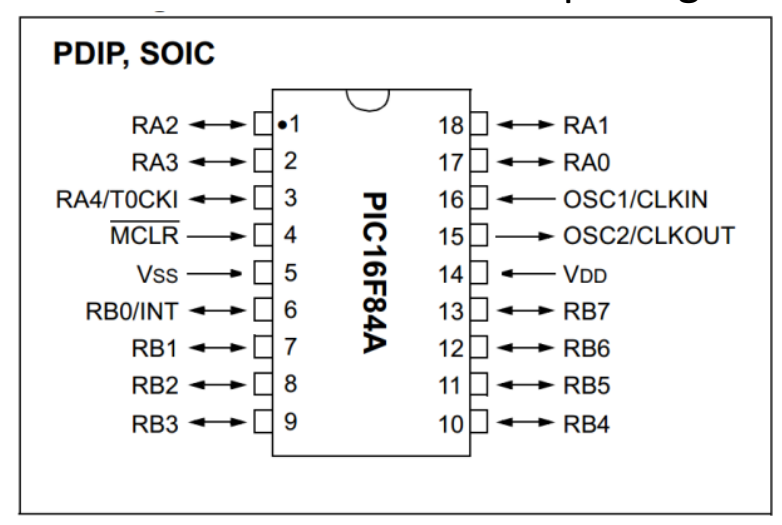

Fig 2: PIC16F84A Microcontroller Layout.

Pin functions consist of two pins for clock, one pin for resetting, five for PORTA as I/O ports, eight for PORTB as I/O ports, and two pins for power supply. Table 1 shows the full description of pins: 


\begin{tabular}{|c|c|c|c|}
\hline Pin Name & PDIP No. & I/O/P Type & Description \\
\hline OSC1/CLKIN & 16 & $\mathrm{I}$ & Oscillator. \\
\hline OSC2/CLKOUT & 15 & 0 & Oscillator. \\
\hline MCLR & 4 & $1 / 0$ & $\begin{array}{l}\text { Master Clear (Reset) } \\
\text { input/programming voltage } \\
\text { Input. This pin is an active low RESET } \\
\text { to the device. }\end{array}$ \\
\hline RAO & 17 & 1/O & PORTA is a bi-directional I/O port. \\
\hline RA1 & 18 & $1 / 0$ & \\
\hline RA2 & 1 & $1 / 0$ & \\
\hline RA3 & 2 & $1 / 0$ & \\
\hline RA4/TOCKI & 3 & $1 / 0$ & \\
\hline RBO/INT & 6 & $1 / 0$ & PORTB is a bi-directional $\mathrm{I} / \mathrm{O}$ port. \\
\hline RB1 & 7 & $1 / 0$ & \\
\hline RB2 & 8 & $1 / 0$ & \\
\hline RB3 & 9 & $1 / 0$ & \\
\hline RB4 & 10 & $1 / 0$ & \\
\hline RB5 & 11 & $1 / 0$ & \\
\hline RB6 & 12 & $1 / 0$ & \\
\hline RB7 & 13 & $1 / 0$ & \\
\hline Vss & 5 & $\mathrm{P}$ & Ground reference. \\
\hline VDD & 14 & $P$ & Positive supply for logic and I/O pins. \\
\hline
\end{tabular}

I=Input $\quad O=$ Output $\quad I / O=$ Input/Output $\quad P=$ Power

TABLE 1: PIC16F84A PINOUT DESCRIPTION.

\subsubsection{PORTA, PORTB and TRISA Registers: \\ 2.3.5 Clock Oscillator and Instruction Cycle:}

PORTA is a 5-bit wide, bi-directional port. The corresponding data direction register is TRISA. Setting a TRISA bit $(=1)$ will make the corresponding PORTA pin as input. Clearing a TRISA bit $(=0)$ will make the corresponding PORTA pin as output.

PORTB is an 8-bit wide, bidirectional port. Also, direction controlled by TRISB register in the same way of PORTA.

All port pins have TTL input levels and full CMOS output drivers. S1:15

Microprocessor or microcontroller is a complex electronic circuit, consisting of serial and standard logic. At a great speed, it steps in turn through a series of complex states, each state dependent on the sequence of instructions that are executed. While the details of this process are not visible to us, it is still necessary to provide "clock signal, constantly operate constant frequency square wave wave.The total speed of the microcontroller process depends entirely on this frequency clock.It is not only the processing unit In most microcontrollers, many of 
the basic timing functions are also derived from it, ranging from the counter / timer functions to the serial communication.

In addition, the total power consumption of the controller has a strong dependence on the clock frequency. With high speed The process is much more than the The hungry power of slow speed. The clock circuit is connected to the timing and mass control to drive all chip operations. For applications where precise timing is not required, a simple external resistor and a capacitor network control the internal oscillator frequency (ARSI). Relatively low frequencies (<shaking) are generated with a vertical clock. For more accurate timing, the crystal oscillator is.

In case of latching, the manufacturer recommends using a resistor between the value of $3 \mathrm{~K}$ $100 \mathrm{~K} \mathrm{ohm}$ and above $20 \mathrm{~V}$ for the condenser, the researcher used $10 \mathrm{~K}$ ohm and $22 \mathrm{~V}$ respectively.

\subsection{MikroC Programming}

\section{Language:}

Micro $C$ is a powerful, feature-rich development tool for PIC Micros.

- debugging, code templates, and more).

- Micro-Pro's use of Pick libraries to accelerate significantly in development included: data acquisition, memory, screens,
It is designed to provide the customer with the easiest solution possible to develop embedded system applications without compromising performance or control.

PIC and C fit together well: PIC is the most popular 8-bit chip in the world, used in a wide range of applications, and $\mathrm{C}$, precious to its efficiency, is a natural choice for the development of embedded systems. Microc provides a highly successful game featuring a highly advanced ID, ANSI-compatible interpreter, a wide range of hardware libraries, comprehensive documentation, and many examples ready to run.

2.4.1 MikroC Features: here are some other features of MikroC language:

- Type the C source code using the built-in code editor (code and parameter helpers, code compiler, syntax highlighting, automatic

conversions, communications and others.

- Microcomputer Translator provides a set of useful functions built-in utilities. 
Embedded functionality does not require any header files to be included. You can use it in any part of your project. S2: 141

- Monitor the program structure, variables, and functions in Code Explorer.

- Use the integrated microcode (in-circuit debugger) real-time debugging tool to monitor program execution at the hardware level.

- Check program flow and debug executable logic with integrated software emulator.

\subsubsection{MikroC Instructions Used:} here are some details about instruction and rules used by the researcher in this project:

Binary Constants: All constants starting with $\mathrm{Ob}$ (or $\mathrm{OB}$ ) are taken to be binary. In the absence of any

\subsubsection{Hardware Tools and} Electronic Components: researcher used:

- Microcontroller chip.

- Power regulator 7805 as $5 \mathrm{~V}$ regulated power supply.

- Two resistors of $10 \mathrm{~K}$ and 420 Ohm.

- 22pf ceramic capacitor.

- main power supply.

- Twelve of LEDs.

- Laptop Computer . overriding suffixes, the data type of a binary constant is derived from its value, according to the rules presented above. For example, Obi 1011 will be treated as short.

(Delay_ms)

(Goto) Statement:

Colon: Use colon (:) to indicate a labeled statement. For example

Start: $\quad \mathrm{x}=0$;

Goto start

(PORTA), (PORTB): assign a value for port related; the value could be in HEX format or BINARY in the form: PORTA=0B00000000

(TRISA), (TRISB): to indicate the state of $1 / O$ ports weather are input or output, assigning (0) to a bit indicates that it is in input state, and (1) indicates that it is in output state

\section{Research Methodology:}

3.1 Equipments, Tools and means used:

- TopWin universal EEPROM Programmer.

\subsubsection{Software:}

- MikroC pro.

- ISIS-Proteus, Version 7 simulator.

- TopWin universal programmer software.

- Windows 7.

3.2. Circuit Diagram and Building the Experiment:

Figure 3 shows the typical diagram of the circuit of the project, 
showing only one LED diode

LEDs

in connected to BO (RBO), and other

the experiment connected adapter with bridge diodes) according to Table 2 . The researcher chose to use the Arcy circuit for clock timing (R1 and C1) because we do not need to High speed and accuracy, that gives us clock frequency less than $1 \mathrm{MZ}$ regulated to $5 \mathrm{~V}$ using $\mathrm{LM} 7805$ regulator U2. R1 used as current flow current through LED (D1).

Researcher builds the circuit approx. Power supply (9V battery or according to Fig3. and Table2:

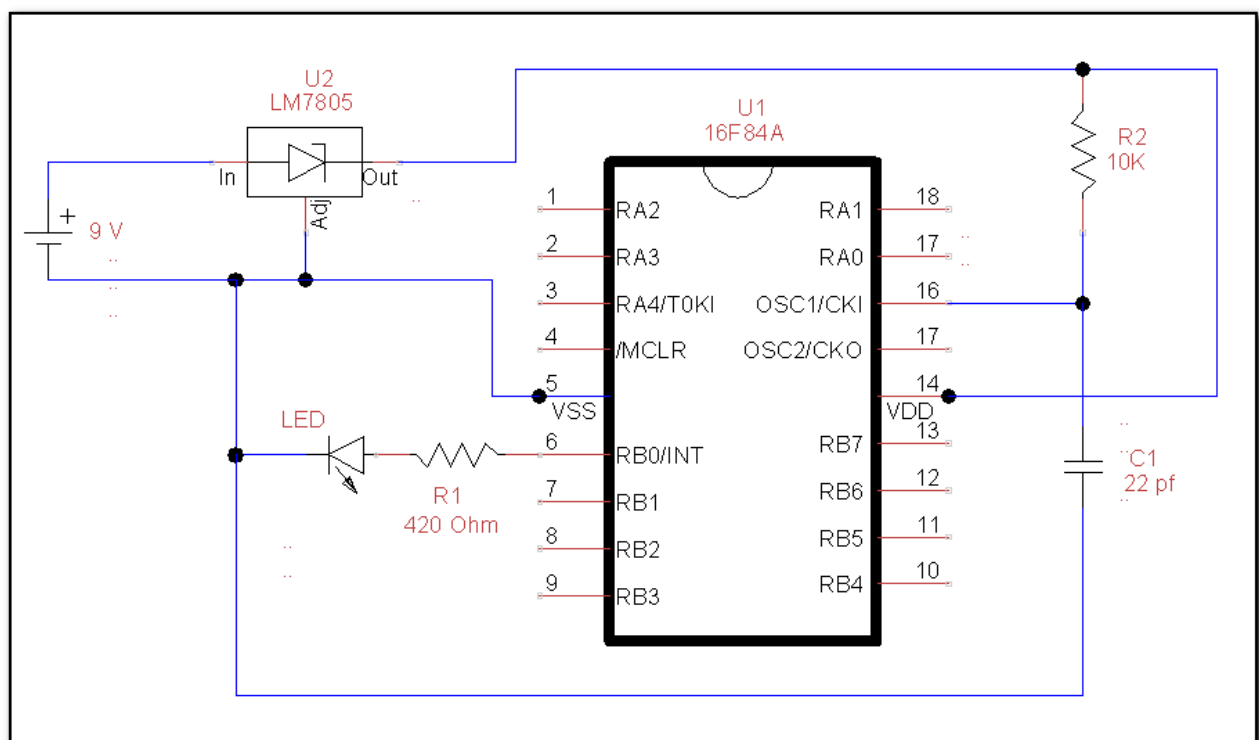

Fig.3: Project Circuit Diagram

\begin{tabular}{|l|l|l|}
\hline Signal target & PORT-bit & Light color \\
\hline Traffic Light 1 & A0 & Red \\
& A1 & Yellow \\
& A2 & Green \\
\hline Traffic Light 2 & A3 & Red \\
& B0 & Yellow \\
& B1 & Green \\
\hline Traffic Light 3 & B2 & Red \\
& B3 & Yellow \\
& B4 & Green \\
\hline Traffic Light 4 & B5 & Red \\
& B6 & Yellow \\
& B7 & Green \\
\hline
\end{tabular}

Table 2: LEDs connection to PORTA and PORTB 
Overall electronic components required were:

\begin{tabular}{|l|l|l|l|}
\hline Item & Type & Value/No. & Quantity \\
\hline U1 & IC & 16F84A & 1 \\
\hline U2 & IC & Lm 7805 & 1 \\
\hline R1 & Resistor & $10 \mathrm{~K}$ Ohm & 1 \\
\hline R2 & Resistor & 420 Ohm & 1 \\
\hline C1 & Capacitor & 22 pf & 1 \\
\hline D1-12 & LED & - & 12 \\
\hline \multicolumn{4}{|c|}{ Table 3: Electronic Components. }
\end{tabular}

\subsection{Programming with mikroC}

\section{Language:}

\subsubsection{Designing and Coding the}

Program: According to the code (see supplement B, P: 14 for code list). Instructions used in the program of the project limited by our needs only, which are:

- TRISB and TRISB: for setting the state of $1 / 0$ ports weather are input or output.

- Delay_ms

for

simulating our project as shown

in Fig.5, test passed successfully. flowchart that we designed (see supplement A, P: 13 for flowchart detail), the researcher used MikroC language for writing

- PORTA and PORTB

- Goto-Label

3.3.2 Debug and Compile the Program: researchers found no syntax error using built in mikroC debugger. Then, the program compiled successfully with no errors.

3.3.3 Test the Program by Simulation: we used SISI-Proteus

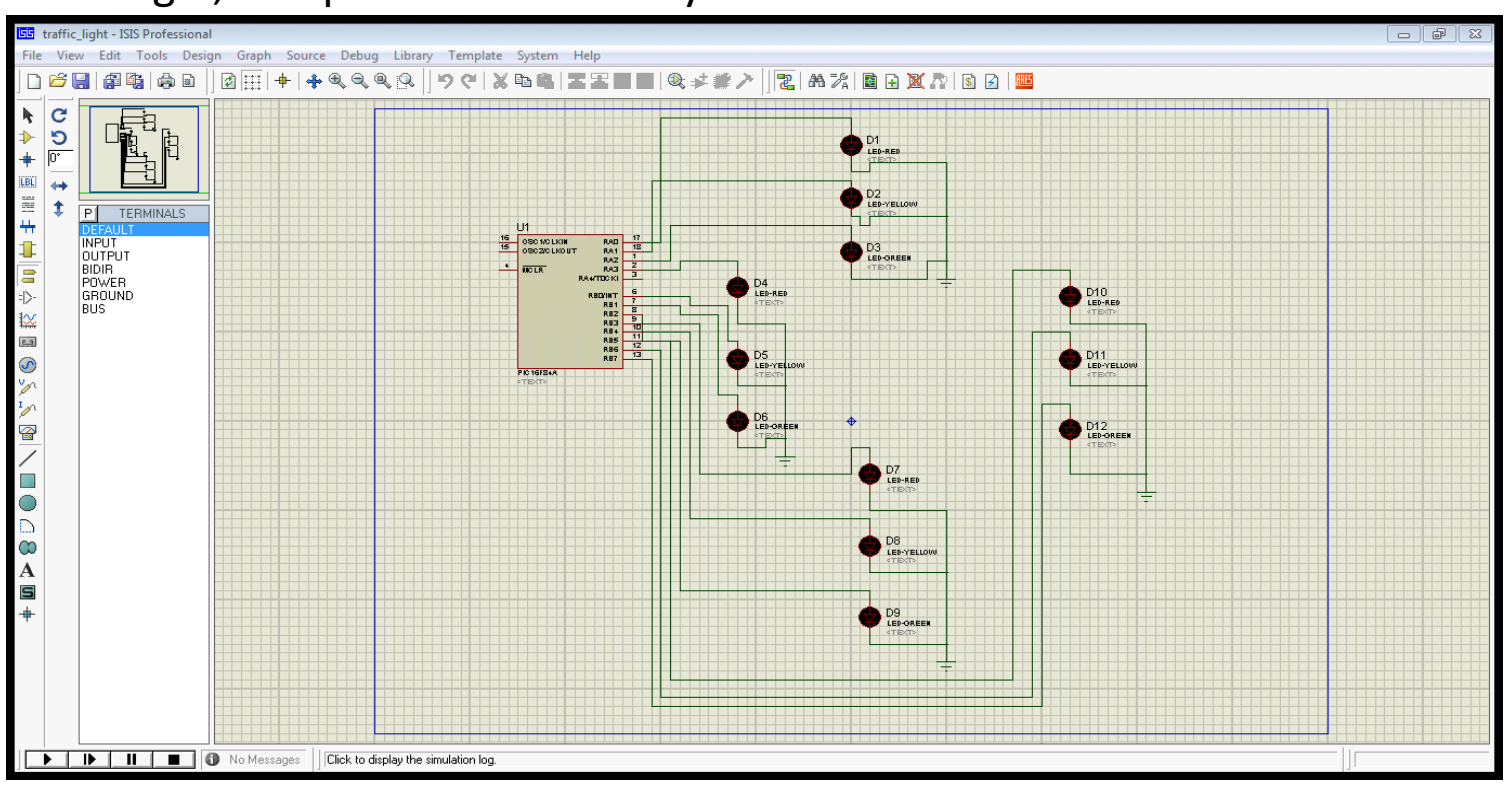

Fig.5 project Simulation using SISI-Proteus Software. 
3.3.4 Transfer the program to the Microcontroller: researchers burned the program to EEPROM of Microcontroller using TopWin universal programmer, the operation done successfully without errors.

\section{Results and discussion:}

According to both theoretical and frameworks, the researcher could built a basic device for controlling traffic light successively, this was just a basic project that could be altered in number of lights, tracks and timing according the situation requirements.

\section{$5 . \quad$ Conclusions}

and

\section{recommendations:}

\subsection{Conclusions:}

According to the results, researcher concludes the following:

- Building a control system using

PIC16F84A

- field.

- Adding more output lights, e.g. for walkers, using more advanced PIC microcontroller, like PIC32MX microcontroller.
3.3.5 Test overall the Project practically:

After that, researcher operates the project in reality; we found that the project is working properly as planned.

Microcontroller is relatively easy.

- Writing code for programming PIC16F84A using MikroC language is not complicated process because of limit instructions we need,

\subsection{Recommendations:}

The researcher recommends the following:

- Developing the project by adding power driver stage for each output in order to operate higher power bulbs to use it in the real 
AL-Qadisiyah Journal of pure Science $\quad$ Vol.23 No. $2 \quad$ Year 2018

\section{Supplements:}

\section{A: Program Flow Chart}

\section{The Program List in MikroC \\ Language:}

Void main ( )

\{

TRISB $=0$ 0B00000000;

TRISA= 0B00000000;

PORTB=0B00000000 ;

PORTA $=0 B 00000001$;

PORTB=0B00100110;

delay_ms(1000) ;

PORTA=0B00000000;

PORTB $=0 B 00000000$;

PORTA=0B00000001;

PORTB $=0 B 00101010$;

delay_ms(500) ;

PORTA $=0 B 00000000$;

PORTB $=0 B 00000000$;

PORTA $=0 B 00001001$;

PORTB $=0 B 00110000$;

delay_ms(1000) ;

PORTA $=0 B 00000000$;

PORTB $=0 B 00000000$;

PORTA $=0 B 00001001$;

PORTB=0B01010000 ;

delay_ms(500) ;

PORTA $=0 B 00000000$;

PORTB $=0 B 00000000$;

PORTA $=0 B 00001001$;

PORTB=0B10000100 ;

delay_ms(1000) ;

PORTA $=0 B 00000000$;

PORTB $=0 B 00000000$;

PORTA $=0 B 00001010$;

$P O R T B=0 B 10000100$;

delay_ms(500) ;

goto loop ;

\}
Loop:

PORTA $=0 B 00001100$;

PORTB $=0 B 00100100$;

delay_ms(1000);

PORTA $=0 B 00000000$;

$P O R T B=0 B 00000000$;

PORTA $=0 B 00000100$;

PORTB $=0 B 00100101$;

delay_ms(500) ;

PORTA=0B00000000 